\title{
Survey Paper on Web Services in IOT
}

\author{
Jyoti L. Khachane ${ }^{1}$, Latika R. Desai ${ }^{2}$ \\ Department of Computer Engineering, D. Y. Patil. Institute of Engineering and Technology, Pimpri, Pune, India
}

\begin{abstract}
In any given context, a number of devices will work together to provide appropriate services to the user. Services are built on the information gathered by IoT devices. The gathered information is raw as well as unstructured in the nature, so there is a need of converting it to meaningful services, User must be made aware of the presence of the services to benefit from it. Further, knowing the presence of a service, users can access services depending on their privileges, roles, preference and requirement. Service discovery will play an important role, as a large number of services could be available in the IoT scenario. Services should be provided only to legitimate users, while others should e prevented from access. Hence, context-aware service discovery and strong access control mechanism will be required in the IoT environment.
\end{abstract}

Keywords: Web Service Discovery, Restful Web Services, Internet of Things, SOAP, Clustering Web Services.

\section{Introduction}

IoT is the unique domain that will works on various things that enables human being to interact with web services as well as helps to obtain the goal of creating a smart world. In this context the research and development challenges to create a smart world. IoT works in all the fields such as making smart city, developing smart transportation system, making smart industry and many other things. The goal of Internet of Things is to connect the people, objects, places at anywhere by introducing various new paradigm. This is the aim of IOT to connect the people, places, things together. A Web service is a type of software application providing platform for IOT based protocols.

Web service is nothing but service Oriented Computing providing better communication among multiple computing platforms, applications, and business parteners. A Web service is an emerging software application that can be identified by a URI, whose interface and bindings are capable of being identified, described and discovered by exchanging XML based messages and supports direct communication with other services via Internet-based protocols. Web service is a part of Service Oriented Computing which enables improved coordination amongst multiple computing platforms, applications, and business partners. Web services are independently developed applications that are exposed as services and interconnected using Web network infrastructure with standards such as XML, UDDI, SOAP and WSDL.

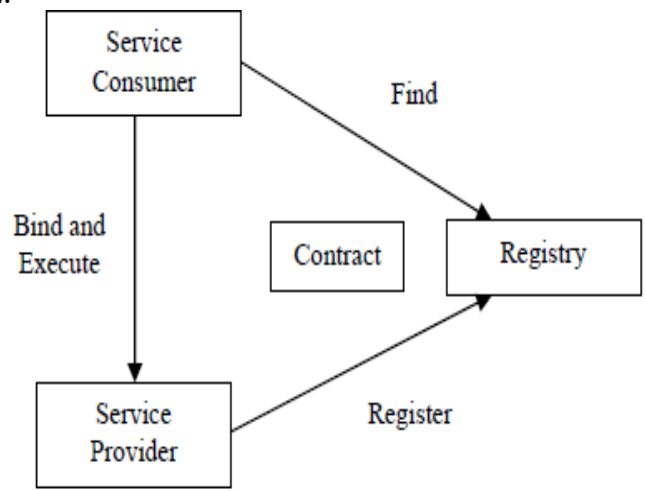

Web services technology is the most promising choice to implement service oriented architecture and its strategic objectives. A Web service is essentially a semantically welldefined abstraction of a set of computational or physical activities involving a number of resources, intended to fulfill a customer need or a business requirement. A Web service could be described, advertised and discovered using standard-based languages, and interacted through Internetbased protocols. Today, two types of Web services are most popular and widely used: SOAP-based Web services and RESTful Web services. The former is based on Web Services Description Language (WSDL) and Simple Object Access Protocol (SOAP), while the latter conforms to the REST architectural principles. The Business Process Execution Language (WS-BPEL) is the current standard language for Web service composition. Its design is centered around the notion of business process used as glue between interacting services. Following the recursive nature of software composition, services are composed into processes, which themselves can be consumed as services. From a syntactical perspective, the service abstraction assumed by BPEL is the one provided by the Web service description language (WSDL). WSDL-based services expose a set of operations using two interaction patterns: synchronous invocation through remote procedure calls, and asynchronous interactions via messaging. These two patterns are directly reflected in the composition mechanisms supported by the BPEL language.

\section{Literature Survey}

a) Zhang, Lili [1] proposes recursive measures of an asynchronous RESTful web service based on the BPEL extention. Various methods are explained in that help to invoke the RESTful web services form the Intenet of Things. A BPEL process is published here for extending BPEL so that performance and speed will be increased.

b) In[2] using wearable body sensors and smart phones smart healthcare system is implemented and designed. Motion Dection Algorithm is introduced here. Sensors can't save the large amount of raw data. To solve this problem, a temporary function in the mote application is implemented. With this function sensor data will be collected by the mote and will be stored in a text file on the access point so all raw data will be saved properly.

\section{Volume 4 Issue 12, December 2015}




\section{International Journal of Science and Research (IJSR) \\ ISSN (Online): 2319-7064}

Index Copernicus Value (2013): 6.14 | Impact Factor (2014): 5.611

c) GSD a SERVICE DISCOVERY PROTOCOL is introduced in [3] having multiple user-controlled parameters which determines bounds of service caching, and discovery request propagation. Concept of hierarchical grouping of services is also introduced here.

d) Autonomic service discovery criteria is discussed in [4]. A prime criteria is defined here that will Be satisfied for an autonomic service discovery. Authors discuss about the survey and comparison of service discovery approaches.

e) Hang $\mathrm{Wu}$ and Chaozhen Guo[6] introduces a method for making use of Ontology Web Language for classifying Web Services as well as to speed up Web Service discovery. By using some matching algorithm like TF-IDF, position \& meaning of Web Service classification that depends on the matching methods for comparing the service name, parameter is determined. JADE is designed in JAVA, formed by many JAVA packages.

\section{Motivation}

In any given context, a number of devices will work together to provide appropriate services to the user. Services are built on the information gathered by IoT (Internet of Things) devices. The gathered information is raw and unstructured in the nature, so there is a need of converting it to meaningful services. Users must be made aware of the presence of the services to benefit from it. Further, knowing the presence of a service, users can access services depending on their privileges, roles, preferences and requirements. Service discovery will play an important role, as a large number of services could be available in the IoT scenario. Services should be provided only to legitimate users, while others should be prevented from access. Hence, context-aware service discovery and strong access control mechanisms will be required in the IoT environment.

\section{Specification of Web Services}

\subsection{SOAP}

SOAP was originally part of the specification that included the Web Services Description Language (WSDL) and Universal Description, Discovery, and Integration (UDDI). It is used now without WSDL and UDDI. Instead of the discovery process described in the History of the Web Services Specification section below, SOAP messages are hard-coded or genereated without the use of a repository. SOAP commonly uses HTTP, but other protocols such as Simple Mail Transfer Protocol (SMTP) may by used. SOAP can be used to exchange complete documents or to call a remote procedure

\subsection{REST}

REST (Representational state transfer) is an architectural style consisting of a coordinated set of architectural constraints applied to components, connectors, and data elements, within a distributed hypermedia system. REST appeals to developers because it has a simpler style that makes it easier to use than SOAP. It also less verbose so that less volume is sent when communicating. REST ignores the details of component implementation and protocol syntax in order to focus on the roles of components, the constraints upon their interaction with other components, and their interpretation of significant data elements.

\subsection{UDDI}

UDDI is defined as "a set of services supporting the description and discovery of businesses, organizations, and otherWeb services providers, the web services they make available, and the technical interfaces which may be used to access those services" by OASIS (The Organization for the Advancement of Structured Information Standards) . UDDI is an industry initiative that enables businesses to publish their services and allows potential users to discover these services.

\subsection{WSDL}

The Web Services Description Language (WSDL) forms the basis for the original Web Services specification. The following figure illustrates the use of WSDL. At the left is a service provider. At the right is a service consumer.

\section{Technical Aspects}

\subsection{Cluster Discovery}

Mobility plays an essential role in future networks, hence end users should be be able to benefit from the services offered by the premises/infrastructure where they are moving around. Cluster discovery is the first step to make end users aware of the services offered by a cluster of IoT devices. Energy consumption is a major parameter for cluster discovery, as the discovery mechanism should be frequently executed. Bluetooth, Bluetooth low energy and Wi-Fi technologies are typically used for device discovery in a mobile ad-hoc environment. In all these types are analyzed and the Bluetooth technology is chosen for device discovery. The eDiscovery algorithm based on Bluetooth technology is proposed in the research to discover the device. The simulation results show the efficiency of the eDiscovery algorithm over existing ones. Beacon stuffing methods for device discovery based on Wi-Fi technology. The scorebased scanning approach is proposed in this work to make energy efficient device discovery.

Most of the device discovery mechanisms discussed in the literature are working on the MAC layer, however, for middleware services, these approaches are not suitable to apply. To overcome this limitation three algorithms i.e. a connectivity-based dynamic algorithm, a policy-based scalable algorithm and a window-based broadcasting algorithm. After discovering the device, the user must provide his/her preferences and requirements through an interface to benefit from services offered by the cluster of devices. Hence, there is a need of energy efficient device discovery and a user guide to provide the users requirements and to discover and access the services. The research work 


\section{International Journal of Science and Research (IJSR) \\ ISSN (Online): 2319-7064}

Index Copernicus Value (2013): 6.14 | Impact Factor (2014): 5.611

will focus on, to design an energy efficient device discovery algorithm.

\subsection{Service Discovery}

In the IoPTS scenario, there may be a large number of services will be available at specific location, however the user should only get relevant services as per his/her requirements. Service discovery is the process by which a user can identify services of his interest. It involves three roles: service provider, service requester and match maker. The service provider uses a published protocol to advertise the services that it can provide, the user/service requester uses a query protocol to request the service of his interest, and the matchmaker finds the service among all available services, which closely match with the user's preference.

\section{Conclusion \& Future Work}

Grouping the web services required by user as well as their discovery is an important issue in IoT scenario. In the future, home automation, smart cities, intelligent transport and ehealth in such domain IoT applications can be developed. There are large number of devices that can sense the activity that are happening in the surrounding to provide services to the end users. Most of the IoT devices are capable of sensing environmental parameters but do not have the intelligence to give proper response depending on the sensed information. Hence, it is necessary that the IoT devices should be grouped in clusters. Users must be made aware of the presence of cluster to benefit from the services offered by it, and hence there is need of a cluster discovery mechanism. As the energy consumption is the major parameter for cluster discovery. Hence, a lightweight cluster discovery mechanism is required.

\section{References}

[1] Zhang, Lili, et al. "Research on IOT RESTful Web Service Asynchronous Composition Based on BPEL." Intelligent Human-Machine Systems and Cybernetics (IHMSC), 2014 Sixth International Conference on. Vol. 1. IEEE, 2014.

[2] Gao, Ruiling, et al. "Web-based motion detection system for health care."Computer and Information Science (ICIS), 2015 IEEE/ACIS 14th International Conference on. IEEE, 2015.

[3] Chakraborty, Dipanjan, et al. "Toward distributed service discovery in pervasive computing environments." Mobile Computing, IEEE Transactions on 5.2 (2006): 97-112.

[4] Rambold, Michael, et al. "Towards autonomic service discovery a survey and comparison." Services Computing, 2009. SCC'09. IEEE International Conference on. IEEE, 2009.

[5] Rong, Wenge, and Kecheng Liu. "A survey of context aware web service discovery: from user's perspective." Service Oriented System Engineering (SOSE), 2010 Fifth IEEE International Symposium on. IEEE, 2010.
[6] Wu, Hang, and Chaozhen Guo. "The research and implementation of Web Service classification and discovery based on semantic." Computer Supported Cooperative Work in Design (CSCWD), 2011 15th International Conference on. IEEE, 2011.

[7] Guinard, Dominique, et al. "Interacting with the soabased internet of things: Discovery, query, selection, and on-demand provisioning of web services."Services Computing, IEEE Transactions on 3.3 (2010): 223-235.

[8] Im, Janggwan, Seonghoon Kim, and Daeyoung Kim. "IoT mashup as a service: Cloud-based mashup service for the internet of things." Services Computing (SCC), 2013 IEEE International Conference on. IEEE, 2013.

[9] L. Atzori, A. Iera, and G. Morabito, The Internet of Things: A Survey, Elsevier Computer Networks, 2010. 